\title{
Characterization of ZnSe nanocrystals grown by vapor phase epitaxy
}

\author{
V.V. Tishchenko \\ Institute of Physics National Academy of Sciences of Ukraine, 46 Nauki Ave., Kiev 03028, Ukraine \\ E-mail: vvti@iop.kiev.ua
}

A.V. Kovalenko

Dnepropetrovsk National University, 13 Naukova Str., Dnepropetrovsk 49050, Ukraine

Received January 31, 2006

\begin{abstract}
This paper reports the application of scanning electron microscopy, x-ray diffraction, and photoluminescence techniques for characterization of ZnSe nanocrystals grown on GaAs (100) substrate from the vapor phase. The applied characterization techniques show the evidence for coexistence of two sets of nanocrystals with rather different characteristic sizes. In addition, the lowest energy levels of spherically shaped nanocrystals are calculated in the framework of the effective-mass approximation and compared with photoluminescence data.
\end{abstract}

PACS: 81.07.Bc, 81.15.Kk, 78.67.Bf

Keywords: nanocrystals growth, semiconductor nanostructures.

In the last ten years the field of semiconductor nanostructures (SNS) has known a strong development owing to demands in advanced optoelectronic devices, chemical sensors, or even biological labels. A comprehensive up-to-date review on SNS applications can be found in Ref. 1.

Semiconductors that attract the most attention are elemental ( $\mathrm{Ge}, \mathrm{Si}$ ) ones as well as III-V and II-IV compounds. In the pursuit of higher and higher quality and lower fabrication costs, a variety of physical and chemical techniques have been tested in regard to their capability to produce the above materials in nanoscale size and with properties requested from industries. The full list of these diverse techniques existing in a variety of modifications would span a volume. Nevertheless, two major groups can be distinguished among all efforts to produce the SNS: synthesis in colloidal solutions [1,2] and gas-phase synthesis [3-5]. Very challenging strategies in the SNS field are nanoscale lithography [6,7] and biogenic approach $[2,8]$. Each of the techniques used has its own advantages and drawbacks and obviously produce different results.

This work reports characterization of $\mathrm{ZnSe}$ nanocrystals produced by the technique that utilizes the gas-phase synthesis route involving evaporation, nucleation, and growth. The growth technique used is a variant of vapor phase epitaxy (VPE) that uses no alien substances except extremely pure hydrogen, which plays the role of a carrier for transfer of the vapor of the source material to the deposition zone. This technique facilitates the growth of really uncontaminated ZnSe as opposed to the previously reported VPE route utilizing reaction between vapors of $\left(\mathrm{CH}_{3}\right) \mathrm{Zn}: \mathrm{N}\left(\mathrm{C}_{2} \mathrm{H}_{5}\right)$ and $\mathrm{H}_{2} \mathrm{Se}$ in a counterflow jet reactor [9]. For many years the $\mathrm{ZnSe-based} \mathrm{nano-}$ structures have been the focus of much attention for applications in all solid-state blue and green lightemitting devices. For such applications the purity of working media is of great importance.

For this study a series of samples with $\mathrm{ZnSe}$ nanocrystals atop GaAs(100) substrates were prepared by VPE and assessed by scanning electron microscopy (SEM), x-ray diffraction, and low-temperature photoluminescence (PL). Only substrates with the prevalent grain size around $0.2-0.3 \mathrm{~mm}$ were used. This was assessed by x-ray diffraction topography.

A well-polished, $1 \times 1 \mathrm{~cm}$ GaAs substrate was mounted in a horizontal cylindrical quartz reactor with three axial channels for the vaporization of source ma- 
terials. The reactor was equipped with PC controlled gas delivery system that included automated valves and rotameters. The vaporized materials were transported to the low-temperature deposition zone by forced transport in a stream of high-purity hydrogen purified with palladium membrane cells. A fourth input channel provided means for a further flow of hydrogen to secure the best mixing of source vapor. The source material was high-purity ZnSe powder (Merck) vaporized at $975{ }^{\circ} \mathrm{C}$. Corrections to the stoichiometry were made by the use of evaporation of elemental sources of $\mathrm{Zn}$ and Se. The source-substrate distance was as long as $17 \mathrm{~cm}$. As the apparatus provided for a large $\left(\sim 250^{\circ} \mathrm{C} \cdot \mathrm{cm}^{-1}\right)$ temperature gradient in the deposition zone, a critical factor affecting the result of growth was the verticality of the substrate mounting in the reactor. To preserve this, a special substrate holder was constructed and, in addition, only substrates with variation in plane less than $2 \mu \mathrm{k} \cdot \mathrm{cm}^{-1}$ were used. The temperature of growth was controlled with accuracy of $\pm 1{ }^{\circ} \mathrm{C}$ in the range of $170-250{ }^{\circ} \mathrm{C}$. The reactor used has previously produced $\mathrm{ZnSe}$ based low-dimensional structures of high quality [10].

It should be stated now that the streamline flow of $\mathrm{ZnSe}$ vapor used for synthesis in [10] is not favorable for the production of nanocrystals. Instead, this work exploited a quite different synthesis route, viz., a shock supply of strongly supersaturated $\mathrm{ZnSe}$ vapor into the relatively cool $\left(190-200^{\circ} \mathrm{C}\right)$ reactor zone just before the substrate. This shock supply was secured by relatively high rate $\left(\sim 1.5 \mathrm{~L} \cdot \mathrm{min}^{-1}\right)$ of hydrogen flow along the reactor as well as by a specially designed PC program that manipulated electromagnetic valves (controlling both hydrogen inlet and exhaust) of the gas delivery system. In this case the $\mathrm{ZnSe}$ growth occurs in extremely nonequilibrium conditions, yielding structures for which a typical SEM image is shown in Fig. 1.

The image in Fig. 1 shows in different gradations of gray the variously sized nanoscale objects atop a GaAs substrate, presented in pure black color. To have better fillings of these objects the image from Fig. 1 was analyzed by edge-detection software. The software output is shown by Fig. 2. It is interesting to note that image in Fig. 2 shows some «big» objects (with sizes of a few tens of nanometer) that are also found in Fig. 1, indicating a high reliability of the image processing. At the same time it is clearly seen from Fig. 2 that these «big» objects with nearly spherical shape consist of massive numbers of much smaller crystallites (referred to below as nanocrystallites) with sizes of a few nanometers. (A hint of this can be also found in Fig. 1.) In addition, from the careful examination of booth Fig. 1 and Fig. 2 one can notice

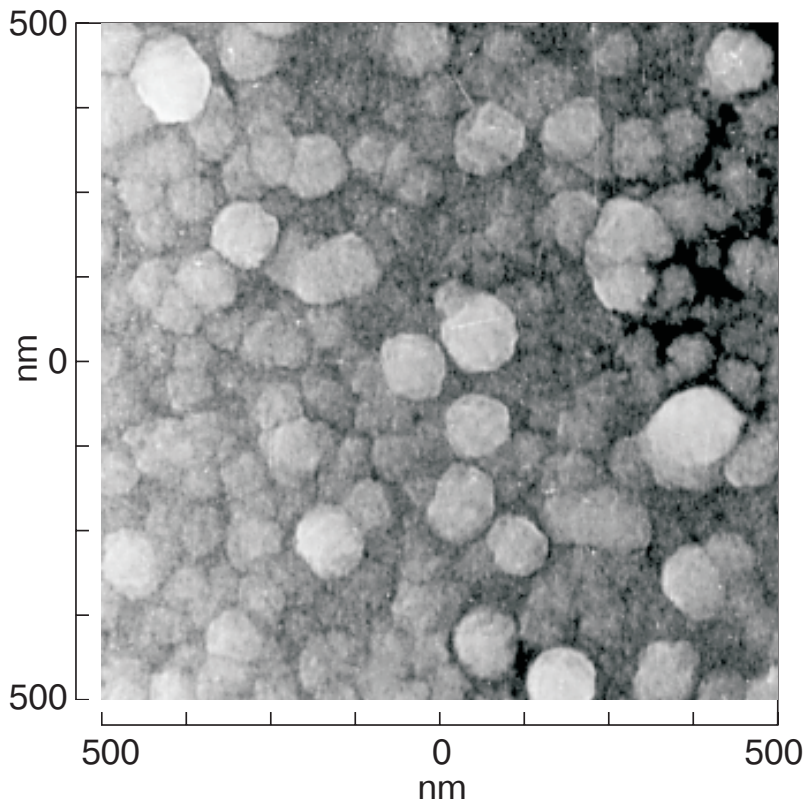

Fig. 1. SEM (Hitachi S-510) image of ZnSe nanocrystals on GaAs substrate.

that these nanorystallites are randomly scattered over all the substrate surface. Apparently they were synthesized at the first stages of the growth procedure. The appearance of big objects evidences that these nanocrystallites have a trend for further aggregation, and their spherical shape gives a hint that such processes took place in the reactor but outside the substrate.

To determine the average size of nanocrystallites, the $\mathrm{x}$-ray diffraction patterns (XRDP) from grown samples were measured in $\theta-2 \theta$ configuration $(\theta$

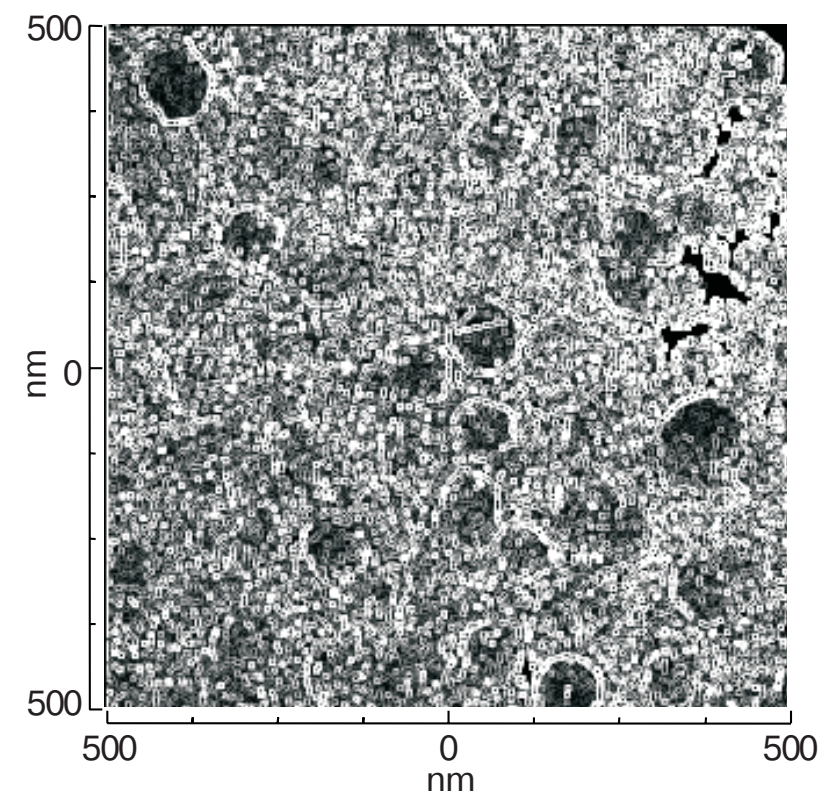

Fig. 2. The result of software processing of the SEM image shown in Fig. 1. 


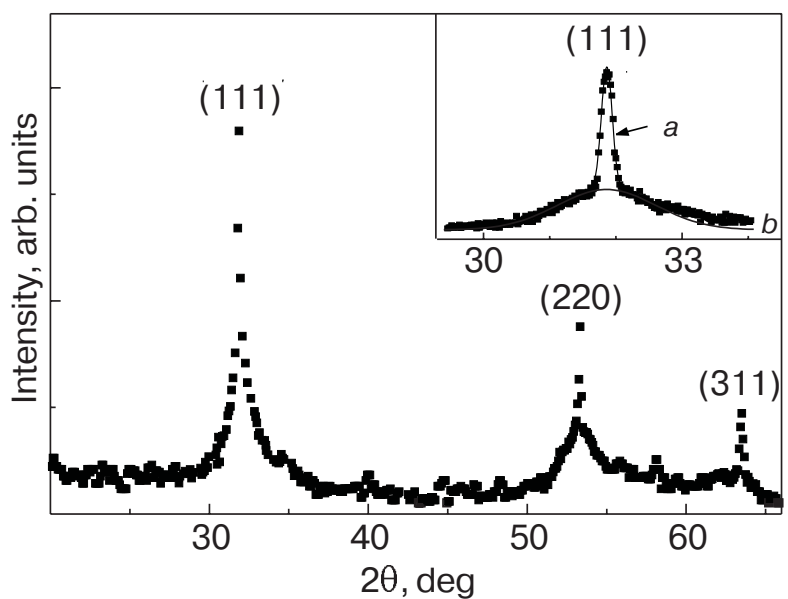

Fig. 3. XRDP of ZnSe nanocrystals on GaAs substrate. Note in the inset the presence of the two Gaussian fits (marked as $a$ and $b$ ) under the (111) ZnSe reflection.

stands for the incidence angle) using DRON-2 diffractometer (upgraded with PC control and data acquisition) that utilized $\mathrm{CoK}_{\alpha}$ radiation $(\lambda=$ $=1.7902 \AA$ ). Figure 3 shows the XRDP for the same sample as in Fig. 1. The remarkable thing about observed XRDP is that it displays clearly three principal diffraction peaks centered at $31.86,53.17$, and $63.30^{\circ}$. The positions of these peaks are in good agreement with the reflections from (111), (220), and (311) crystallographic planes of the cubic modification of $\mathrm{ZnSe}$. The observation of three peaks means that synthesized crystallites of $\mathrm{ZnSe}$ are randomly oriented. This may indicate that these crystallites were collected on the substrate after their formation. Indeed previous experiments have shown that at straight deposition and epitaxial growth on a GaAs(100) substrate the resulting ZnSe structures were (100)-oriented $[10,11]$.

What is more remarkable than the fact that the XRDP of the investigated samples contains reflections from different crystallographic planes of $\mathrm{ZnSe}$ is that these reflections are broadened out and their broadening is not elemental. Roughly each of the observed reflections can be decomposed into two components at the same position: a very narrow component and a broad one. The inset in Fig. 3 shows the decomposition of the observed (111) reflection into two Gaussian curves with doubled standard deviations $(2 \sigma)$ of around 1,487 and $0,165^{\circ}$, respectively.

The question arises why the observed $\mathrm{x}$-ray reflections are so complicated, each of them being a superposition of broad and narrow components with rather different full width at half maximum (FWHM). Obviously it suggests that two distinct sets of $\mathrm{ZnSe}$ nanocrystallites coexist on the same substrate. Accordingly two sets of $x$-ray signals from the sample are observed at the same Bragg angle. In other words, the size distribution of relevant $\mathrm{ZnSe}$ nanocrystallites on GaAs substrate is bimodal, with two strongly differing characteristic sizes that determine the widths of relevant components of the same reflection. The set of crystallites with the smaller characteristic size (further referred to as the $S$-set) contributes to the broader component ( $S$-component), while the set of crystallites with the larger characteristic size ( $L$-set) donates the narrow component ( $L$-component). To distinguish between these sets of crystallites, a convention was established that physical values with $S$ as a superscript will always represent the $S$-set; analogously, $L$ as a superscript will stand for the $L$-set.

To extract information about the size of the nanocrystallites from the XRDP, a separation of the peak broadening caused by the diffractometer from the sample-generated (intrinsic) broadening was performed first. Assuming a pure Gaussian shape for both intrinsic and instrumental profile, one can write [12]:

$$
\sigma_{\text {int }}^{S, L}=\sqrt{\left(\sigma_{m}^{S, L}\right)^{2}-\sigma_{i}^{2}}
$$

where $\sigma_{\text {int }}^{S, L}, \sigma_{m}^{S, L}$, and $\sigma_{i}$ are the standard deviations for the intrinsic profiles, measured profiles, and instrumental profile, respectively.

The instrumental broadening was determined from the (400) reflection (measured with the finest available step of $0.01^{\circ}$ ) of the GaAs substrate at $78.58^{\circ}$ (not shown in Fig. 3). As the substrate has grains large enough to eliminate the size-broadening effect, it generates diffraction line broadening which is affected only (by surmise) by the instrumental contribution. The fit of a Gaussian function to the (400) peak yielded $2 \sigma_{i} \approx 0,066^{\circ}$. As this value is considerably less than those obtained from the fitting of Gaussians to the $S$-components in XRPD, the instrumental broadening correction of these components was disregarded, and only the $L$-components were corrected.

It is well known that besides the crystallite size, the shape of the Bragg reflexes conveys information of the nonuniform interior strain. The approaches used to extract both strain and size effect from the distortion of the Bragg peaks generally involve fitting mathematical functions (e.g., Lorentzian, Gaussian, Voigt, etc.) to the shapes of observed reflections, and then obtaining desired information according to theoretical models assumed to be correct for the investigated materials [13-15]. With many successful results available, the objective of this research was not to compete with these results on the basis of choosing the appropriate functions and / or fitting procedure, but to present the problems of nanocrystal growth from the perspective of VPE technique. Therefore for the sake of simplicity we took no notice of the possible strain con- 
tribution into broadening of observed x-ray reflections and assumed that only size effect was appreciable. More likely than not this assumption did not affect significantly the results obtained for the $S$-set of nanocrystallites due to the considerable broadening of $S$-components in XRDP. The $L$-components are more than ten times narrower than $S$-component and as such should be very sensitive to the strain effect. However, it is evidenced below that the characteristic size estimated on the assumption of minor strain contribution to the $L$-components does not vary significantly from peak to peak, and hence the above assumption is not too far from truth. Nevertheless the last statement will be studied somewhere else by using a combination of precision x-ray diffraction and Raman spectroscopy.

With above assumption the FWHM of the intrinsic diffraction line $\left(\delta^{S, L}(2 \theta)=2 \sigma_{\text {int }}^{S, L} \sqrt{2 \ln 2}\right.$, in radians $)$ corresponds to crystallite size following the Debye-Scherrer formula [16]:

$$
\delta^{S, L}(2 \theta)=\frac{K \lambda}{H_{h, L, l}^{S, L} \cos \theta},
$$

where $H_{h, k, l}^{S, L}$ stands for a volume-weighted column height of crystallite in the [hkl] direction, and $K$ is a somewhat arbitrary geometry-dependent constant whose value is typically between 0.85 and 1.00 . Tabulated values of $K$ for some cubic crystals can be found in Ref. 17.

On the basis of Eqs. (1), (2) the characteristic size for both the $S$-set and $L$-set of ZnSe nanocrystallites can be calculated from the fitting of Gaussian functions to the observed reflections. The best fit is achieved with values listed in Table 1 . Table 1 does not offer data for the $S$-set in the [311] direction. For the reason that the noisy signal in this direction prevents precise fitting, the relevant data were omitted to be on the safe side. Because the choice of the best value of the $K$ factor in Eq. (2) is something of a mystery under the condition that the nanocrystallites shape is not known a priori (the values most frequently used in the literature are $0.89,0.93$ and 1 ), Table 1 gives not the exact numbers for $H_{h, k, l}^{S, L}$ but its range obtained with the limit values of the $K$ coefficient. Despite such an approximate answer to the question of the size of the nanocrystals it facilitates the «guesstimates» relevant to their shape. Namely, from Table 1 an identical trend is visible for both the $L$-set and $S$-set of nanocrystallites: their characteristic size does not depend on the observation direction. On the one hand, it suggests negligibly small interior strains present in these crystallites, and, on the other hand, it hints at a spherical shape of these objects.
Table 1. Results of Gaussian fitting and Scherrer analysis. In the 2nd and 3rd columns are mean values for the set of 10 samples. The 4 th and 5 th columns give values calculated with $K=0.89$ (lower bound) and $K=1$ (upper bound).

\begin{tabular}{c|c|c|c|c}
\hline \hline$h k l$ & $2 \sigma_{\text {int }}^{L}, \operatorname{deg}$ & $2 \sigma_{\text {int }}^{S}, \operatorname{deg}$ & $H_{h, k, l}^{L}, \mathrm{~nm}$ & $H_{h, k, l}^{S}, \mathrm{~nm}$ \\
\hline \hline 111 & $0.152 \pm 0.017$ & $1.489 \pm 0.121$ & $53.0-59.6$ & $5.4-6.0$ \\
220 & $0.156 \pm 0.02$ & $1.693 \pm 0.29$ & $55.6-62.4$ & $5.1-5.8$ \\
311 & $0.162 \pm 0.018$ & - & $56.2-63.17$ & - \\
\hline \hline
\end{tabular}

For spherically shaped crystallites of radius $R$ one can write [13]:

$$
<H_{h, k, l}^{S, L}>=\frac{3}{2} R^{S, L},
$$

in which the brackets indicate the averaging of data with different Miller indices. From the data presented in Table 1 and Eq. (3) it was found that characteristic radii for two coexisting sets of $\mathrm{ZnSe}$ nanocrystallites resulting from the VPE technique used were $R^{L} \approx 36.6-41.2 \mathrm{~nm}$ and $R^{S} \approx 3.5-4.0 \mathrm{~nm}$.

It should be mentioned here that tens-of-nanometer sized agglomerates (nanoparticles) of $\mathrm{ZnSe}$ with fine structure that are clearly distinguished in Fig. 1 and Fig. 2 do not contribute to the $L$-components of the $\mathrm{x}$-ray reflections, while on the contrary, their finest parts of a few nanometers in size give the $S$-components.

From this point we proceed with the results of the PL measurements. They were obtained on samples mounted inside an optical cryostat operating at $77 \mathrm{~K}$. The PL was excited by the $325 \mathrm{~nm}$ line of a $10 \mathrm{~mW} \mathrm{cw}$ $\mathrm{He}-\mathrm{Cd}$ laser and analyzed by a computer-controlled double monochromator with photon counting detection.

Figure 4 shows the photoluminescence data for the same sample as in Fig. 1. Two broad PL bands with maximum emission intensities located at $2.854 \mathrm{eV}(S)$ and $2.797 \mathrm{eV}(L)$ are clearly seen, in agreement with an assumption of two coexisting nanocrystallites sets on the same substrate. That is, we argue that the $S$ band in the PL spectrum in Fig. 4 originates from the transitions relevant to the $S$-set of ZnSe nanocrystallites, while the $L$ band is related to the transitions from the $L$-set. The ratio of integrated intensities of these PL bands can be regarded as simply the ratio of volumes for two sets of ZnSe nanocrystallites, showing that the $S$-set is dominant. (Similar explanation applies for the ratio of the integrated intensities of $S$-components and $L$-components of XRDP in Fig. 3.)

The energy of the maximum emission intensity for the $L$ band agrees closely with the excitonic energy 


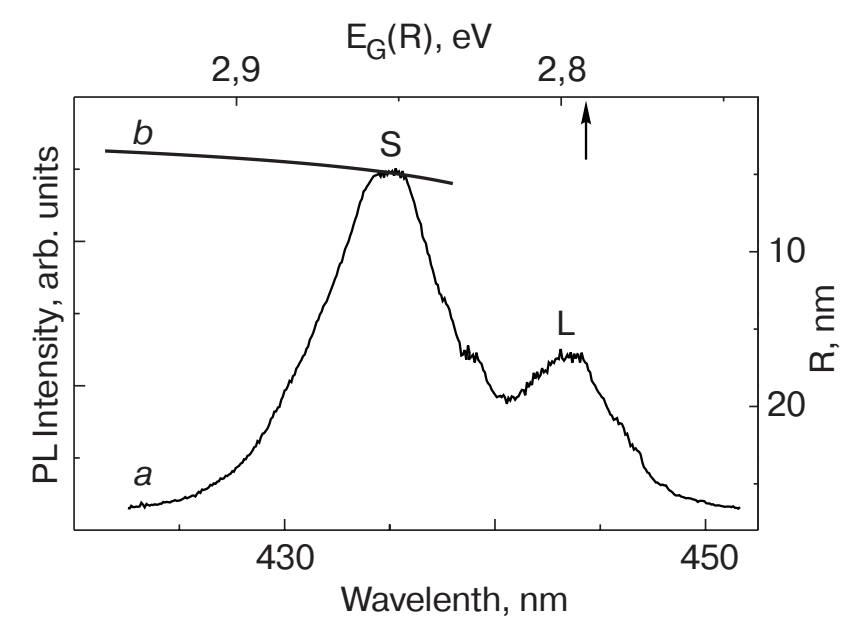

Fig. 4. PL spectrum (a) of ZnSe nanocrystals on GaAs substrate at $77 \mathrm{~K}$ in relation to the calculated band gap $E_{G}(R)$ (presented by line $b$ ) of the spherical nanocrystals with radius $R$. The spectrum has been normalized to the intensity of the $S$ band. The arrow indicates the value of the excitonic energy gap for bulk ZnSe at $77 \mathrm{~K}$.

gap (2.791 eV, Ref. 18 ) of the bulk ZnSe at $77 \mathrm{~K}$ but is blue shifted by $43 \mathrm{meV}$ for the $S$ band. We now compare this observation with available theoretical models describing in the framework of the effective mass approximation the size effect on the high-energy shift of the ground state of the electron - hole system.

For the $L$-set of nanocrystals one should consider that $R^{L} a_{B}^{-1} \sim 10\left(a_{B} \sim 50 \AA\right.$ is the effective Bohr radius of the free exciton in bulk ZnSe [19]) and, thus one can expect the following $R^{L}$-dependence of the relevant shift [20]:

$$
\Delta E^{L}=\frac{\hbar^{2} \pi^{2}}{2 M\left(R^{L}-\eta a_{B}\right)^{2}},
$$

in which $M$ is the sum of the effective masses of electron $m_{e}^{*}$ and hole $m_{h}^{*}$, and $\eta$ is a constant whose numerical value is a function of $m_{h}^{*} / m_{e}^{*}$. As was noted in Ref. 20, the term $\eta a_{B}$ in Eq. (4) takes into account the excitonic dead layer, that is the nearsurface region of a nanocrystal that cannot be reached by the center of mass of an exciton due to the finite size of this quasiparticle. Using $m_{e}^{*}=0.17 m_{0}, m_{h}^{*}=$ $=0.60 m_{0}[21]$, and $\eta=1.1[20]$, the calculated $\Delta E^{L}$ is around $1 \mathrm{meV}$, showing that for such «big» nanocrystals as the $L$-set the effect of quantum confinement is small. One can see that this coincides within a few millielectron-volts with experimental data presented by Fig. 4. However a small discrepancy exists, and most likely this comes from the size determination.

In contrast for the $S$-set of nanocrystals we have $R^{S} a_{B}^{-1} \sim 1$. In this case to simulate the size dependence of the band gap $\left(E_{G}\right)$ of the spherically shaped semiconductor one can use the following expression [20]:
$E_{G}(R)=E_{0}+\frac{h^{2} \pi^{2}}{2 R^{2}}\left(\frac{1}{m_{e}^{*}}+\frac{1}{m_{h}^{*}}\right)-\frac{1,786 e^{2}}{4 \pi \varepsilon_{0} \varepsilon R}-0.248 E_{\mathrm{Ry}}^{*}$,

where the first term on the right side stands for the band gap of the bulk semiconductor crystal; $\varepsilon$ and $E_{\mathrm{Ry}}^{*}$ are the dielectric constant of semiconductor and effective Rydberg energy of the free exciton, respectively (the relevant numerical values for $\mathrm{ZnSe}$ are 8.1 and $20 \mathrm{meV}$ [21]). The fourth term in Eq. (5) is due to the spatial correlation between the electron and the hole.

The calculated relevant dependence of band gap based on Eq. (5) is plotted as line $b$ in Fig. 4. Clearly, it would be necessary to compare this calculation and PL excitation measurements. Not having access to PL excitation data, we state that the above calculation cannot precisely reproduce the PL results for the $S$-set. Besides the size determination, the discrepancy may be caused by the presence of a Stokes shift and/or failure of the effective-mass approximation for relatively small nanocrystals.

In conclusion, we have described a simple technique for vapor phase synthesis of ZnSe nanocrystals on a GaAs substrate. The design of the VPE reactor enables growth at relatively low temperatures. The nanocrystals were characterized by x-ray diffraction, SEM, and low-temperature photoluminescence techniques.

There remains a question about the nature of the bimodal size distribution of $\mathrm{ZnSe}$ nanocrystals. It is clear that the simplest mechanism that leads to formation of nanocrystals should resemble the process described in Ref. 9. When the hot vapor of $\mathrm{ZnSe}$ enters a cooled zone of reactor it becomes supersaturated and undergoes a fast condensation, resulting in singlecrystalline nuclei. The nuclei with radii larger than a critical size propagate along the reactor tube and undergo further growth by absorbing gaseous constituents. The nanocrystallites and their aggregates formed via coagulation are collected on the GaAs substrate. Assuming that the growth of nanocrystallites is monotonic with time, one would expect them to have a monomodal size distribution. However, this is not the case, and applied characterization techniques indicate the synthesis of nanocrystals with a size distribution that follows a more complicated law. Obviously, to find the solution to this problem, a refined analysis is needed. In reality the gas phase composition is not uniform. It contains such chemicals as $\mathrm{H}_{2}, \mathrm{ZnSe}, \mathrm{Zn}$, $\mathrm{H}_{2} \mathrm{Se}$, and $\mathrm{Se}$, and, hence, one may include in the analysis whatever additional set of reversible chemical reactions are desired. In addition this analysis (if any) must consider the strong nonequilibrium state of the 
vapor mixture in reactor and/or extra nucleation/growth on the substrate.

1. A. Nabok, Organic and Inorganic Nanostrustures, Artech House, Boston - London (2005).

2. P. Alivisatos, Pure Appl. Chem. 72, 3 (2000).

3. F. Guffarth, R. Heitz, A. Schliwa, O. Stier, N.N. Ledentsov, A.R. Kovsh, V.M. Ustinov, and D. Bimberg, Phys. Rev. B64, 085305 (2001).

4. R. Nötzel, Semicond. Sci. Technol. 11, 1365 (1996).

5. S. Rodt, A. Schliwa, K. Pötschke, F. Guffarth, and D. Bimberg, Phys. Rev. B71, 155325 (2005).

6. R. Held, T. Heinzel, A. Studerus, K. Ensslin, and M. Holland, Appl. Phys. Lett. 71, 2869 (1997).

7. R. Held, S. Lüscher, T. Heinzel, K. Ensslin, and W. Wegscheider, Appl. Phys. Lett. 75, 1134 (1999).

8. J. Dutta and H. Hofmann, in: Encyclopedia of $\mathrm{Na}$ noscience and Nanotechnology, H.S. Nalva (ed.), Vol. X, American Scientific Publishers (2003).

9. D. Sarigiannis, J.D. Peck, G. Kiosoglou and A. Petrou, Appl. Phys. Lett. 80, 4024 (2002).

10. A.V. Kovalenko and V.V. Tishchenko, Jpn. J. Appl. Phys. 34, 209 (1995).
11. V.V. Tishchenko, Y.S. Raptis, E. Anastassakis, and N.V. Bondar, Solid Status Commun. 96, 793 (1995).

12. M. Čerňansky, Mater. Struct. 7, 3 (2000).

13. D. Balzar, N. Auderband, M.R. Daymond, A. Fitch, A. Hewat, J.I. Langford, A. Le Bail, D. Louër, O. Masson, C.N. McCowan, N.C. Popa, P.W. Stephen, and B.H. Topa, J. Appl. Cryst. 37, 911 (2004).

14. J. Landford, J. Appl. Cryst. 11, 10 (1978).

15. D. Balzar, J. Appl. Cryst. 25, 559 (1992).

16. A.L. Patterson, Phys. Rev. B56, 978 (1939).

17. A.R. Stokes and A.J. Wilson, Proc. Phys. Soc. 56, 174 (1944).

18. L. Malikova, W. Krystek, F.H. Pollak, N. Dai, A. Cavus, and M.C. Tamargo, Phys. Rev. B54, 1819 (1996).

19. H. Hayashi and Sh. Katayama, Phys. Rev. B39, 8743 (1989).

20. Y. Kayanuma, Phys. Rev. B38, 9797 (1988).

21. G.H. Nelkowski and H.J. Schulz, in: Landolt-Bornstein Numerical Data and Functional Relationships in Science and Technology, O. Madelung, H. Schulz, and H. Weiss (eds.), Group III, Vol. 17, Subvol. b, Springer, Berlin (1982). 